\title{
Quantitative Analysis for Aluminium Alloy Welding Quality based on Current Sample Entropy
}

\author{
Tian Fa LIAO, Xiao Hui WEI, Yu XU \\ School of Electronic information and Electrical engineering, Huizhou University, Huizhou, China
}

\begin{abstract}
This paper proposed a kind of analysis method for the aluminium alloy welding quality based on current sample entropy to solve the difficulties in the quantitative analysis for the welding stability of aluminium alloy. With the decrease of electrical signal stability, the sample entropy mean increased, and the standard deviation of sample entropy increased. The welding stability was evaluated by the product of the sample entropy mean and the standard deviation, and this was verified well by the test.
\end{abstract}

\section{Introduction}

The aluminium alloy was increasingly applied to the fields such as automobile, ships, aerospace, and so on, due to its excellently comprehensive properties. Moreover, the welding played an important role in the industrial production of aluminium alloy. With the development of industrial production, it is needed for the welding to rapidly and accurately evaluate the welding quality.

\section{Quantitative analysis method of welding quality}

\subsection{Analysis system of current sample entropy}

The electrical signals in the welding process can reflect the technological properties and quality of welding. It is helpful to deeply study the arc mechanism to improve the welding technology and quality by analyzing and studying the electrical signals. Experts in welding industry can quantitatively describe the stability in the welding process.

The Takens theorem in the nonlinear system referred to the evolution information of a system in the development of each state component. Namely, the time series of the state component included relevant informations of all the variables related to the dynamical system in order to describe the deep-level dynamic law. In the early 1990s, Pincus proposed the concept of approximate entropy to overcome the difficulty in the solution of entropy in the chaos phenomenon.It can nonnegatively quantitatively describe the nonlinear time series complexity. The larger the generating probability of new pattern in the measure signals was, the more complex the series was, and thus the larger the corresponding approximate entropy was. [1-4]

On account of the poor consistency of approximate entropy, Sample Entropy (SaEn) was put forward by Richman based on the approximate entropy in 2000, which was a new kind of measure method for the time series complexity[5]. It is unnecessary for the SaEn to calculate its own matching value, and its calculation rate was faster. Thus, the approximate entropy error decreased, as well as its accuracy and consistency were better[6].

It is assumed that the initial data were the sequence with $\mathrm{N}$ points, namely, $\mathrm{u}(1), \mathrm{u}(2), \ldots, \mathrm{u}(\mathrm{N})$. When the dimensions of $\mathrm{m}$ and $\mathrm{m}+1$ were embedded, the first $\mathrm{N}-\mathrm{m}$ vectors of data sequence should meet $1 \leq \mathrm{i} \leq \mathrm{N}-\mathrm{m}$, and thus the m-dimensional $\mathrm{X}_{\mathrm{m}}(\mathrm{i})$ was meaningful.

The steps of SaEn algorithm were as follows.

(1) A group of m-dimensional vectors was reconstructed according to the serial number.

$$
X_{m}(i)=|u(i), u(i+1), \cdots u(i+m-1)|
$$$$
(\mathrm{i}=1,2, \cdots, \mathrm{N}-\mathrm{m}+1)
$$

(1)

(2) The distance between $X_{m}(i)$ and $X_{m}(j)$ was defined as the maximal difference between the two groups of corresponding elements.

$$
\mathrm{d}\left|\mathrm{X}_{\mathrm{m}}(\mathrm{i}), \mathrm{X}_{\mathrm{m}}(\mathrm{j})\right|=\max (|\mathrm{u}(\mathrm{i}+\mathrm{k})-\mathrm{u}(\mathrm{j}+\mathrm{k})|)
$$

(3) For each $\mathrm{i}(1 \leq \mathrm{i} \leq \mathrm{N}-\mathrm{m}), \mathrm{N}^{\mathrm{m}}$ (i) referred to the number that $d\left|X_{m}(i), X_{m}(j)\right|$ was less than the given threshold $\mathrm{r}$, and the ratio of $\mathrm{N}^{\mathrm{m}}(\mathrm{i})$ to $\mathrm{N}-\mathrm{m}$ was solved.

$$
\mathrm{B}_{\mathrm{r}}^{\mathrm{m}}(\mathrm{i})=\mathrm{N}^{\mathrm{m}}(\mathrm{i}) /(\mathrm{N}-\mathrm{m})
$$

(4) The mean of $\mathrm{B}_{\mathrm{r}}^{\mathrm{m}}(\mathrm{i})$ was figured out.

$$
B^{m}(r)=\frac{\sum_{i=1}^{N-m} B_{r}^{m}(i)}{N-m+1}
$$

(5) The $(m+1)$-dimensional vector was obtained by adding 1 to the dimension.

*Corresponding author: Email: liaotianfa@163.com 


$$
\begin{aligned}
& X_{m+1}(i)=|u(i), u(i+1), \cdots u(i+m)| \quad \quad(i= \\
& 1,2, \cdots N-m)
\end{aligned}
$$

(6) For the $(m+1)$-dimensional vector, the steps (2), (3) and (4) were repeated, and then the result was:

(6)

$$
\mathrm{B}_{\mathrm{r}}^{\mathrm{m}+1}(\mathrm{i})=\mathrm{N}^{\mathrm{m}}(\mathrm{i}) /(\mathrm{N}-\mathrm{m}-1)
$$

$$
B^{m+1}(r)=\frac{\sum_{i=1}^{N-m} B_{r}^{m+1}(i)}{N-m}
$$

(7) The SaEn corresponding to the sequence theoretically was

$$
\operatorname{SaEn}(m, r, N)=\lim \left\{-\operatorname{In}\left|B^{m+1} / B^{m}(r)\right|\right\}
$$

If $\mathrm{N}$ was a finite value, it can be expressed as

(9)

$$
\operatorname{SaEn}(m, r, N)=-\operatorname{In}\left|B^{m+1} / B^{m}(r)\right|
$$

Hence, there needed three parameters, namely, the embedded dimension $\mathrm{m}$, the given threshold $\mathrm{r}$ and the sample length $\mathrm{N}$, to calculate the $\mathrm{SaEn}$, as well as different $\mathrm{m}$ and $\mathrm{r}$ corresponded to different SaEns.

\subsection{Quantitative analysis analysis of current SaEn}

At present, there lacked relevant theoretical research on the values of embedded $\mathrm{m}$ and given threshold $\mathrm{r}$ of the current SaEn. This paper analyzed the current signals data by using the SaEn as well as analyzed the stability of current signals by comparing the mean of SaEn and the standard deviation of SaEn defined in Equation (9), as shown in Equation (10).

$$
S D(S a E n)=\sqrt{\frac{\sum_{i=1}^{n}\left(x_{i}-\bar{x}\right)^{2}}{n-1}}
$$

Where $\mathrm{SD}(\mathrm{SaEn})$ referred to the standard deviation of SaEn.

\subsubsection{Influence of current signals on SaEn}

The current signals were analyzed by the SaEn algorithm. The current signals of DMIG welding for aluminium alloy were analyzed and selected, the welding parameters were fh, Ip and Ib, and there values were $100 \mathrm{~Hz}, 220 \mathrm{~A}$ and 50A, respectively. For the comparison and analysis, the waveform quality of four groups of current signals was good, better, worse and bad, respectively. Furthermore, the results of SaEn were normalized for comparison. Assuming that the embedded dimendion was 3 , the similar tolerance $r$ was 0.08 , and the sample length $\mathrm{N}$ was 2000, sixteen SaEns for each signal were calculated, and then the curves SaEn1, SaEn2, SaEn3 and SaEn4 were obtained, as shown in Figure 1.

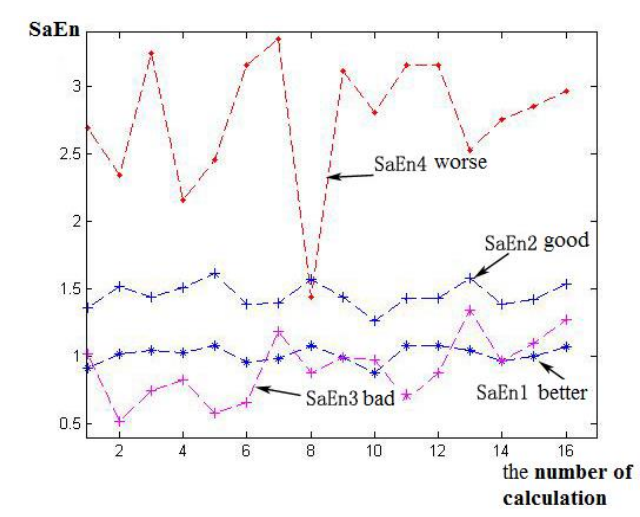

Figure 1 Changes in approximate entropy of different current signals

It can be clearly seen in Figure 1 that the better the waveform quality of electrical signal was, the smaller the SaEn was, and thus the smaller the influence of the number of calculation groups on $\mathrm{SaEn}$.

Equation (10) was used to figure out the standard deviation of $\mathrm{SaEn}$ for four electrical signals. The standard deviations of $\mathrm{SaEn}$ corresponding to four electrical signals from good to bad were 0.00065, 0.00112, 0.00373 and 0.00834 , respectively, and thus following a change law from large to small.

Hence, both the SaEn and the standard deviation of the electrical signals can be used to quantitatively analyze the welding quality. Then, this paper defined the quantitative analysis index CSaEn for the welding quality in Equation (11). Obviously, the smaller the CSaEn was, the better the welding quality was.

$$
\mathrm{CSaEn}=\mathrm{SaEn} \times \mathrm{SD}(\mathrm{SaEn})
$$

\subsubsection{Influence of embedded dimendion $m$ on SaEn}

The electrical signals with better welding quality were used to analyze the influence of embedded dimendion $\mathrm{m}$ on SaEn. The SaEn curves SaEn5, SaEn6 and SaEn7 were obtained in Figure 2 when the given threshold $r$ was 0.08 , the sample length $\mathrm{N}$ was 2000 , the number of calculation groups were 16 , as well as the embedded dimensions were 1, 3 and 5, respectively.

As shown in Figure 2, the shape change of SaEn curve was slightly related to the dimension m. However, the larger the embedded dimendion $\mathrm{m}$ was, the smaller the SaEn was. The standard deviations of SaEn5, SaEn6 and $\mathrm{SaEn7}$ were 0.093, 0.0612 and 0.0593, respectively. 


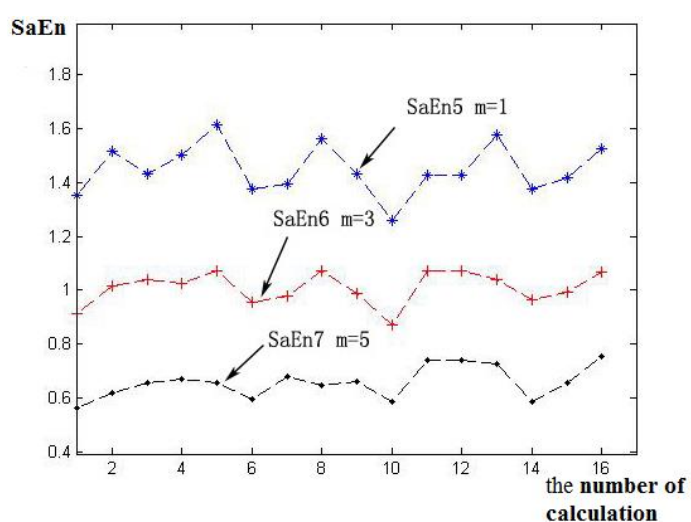

Figure 2 Sample entropy changes with $\mathrm{m}$

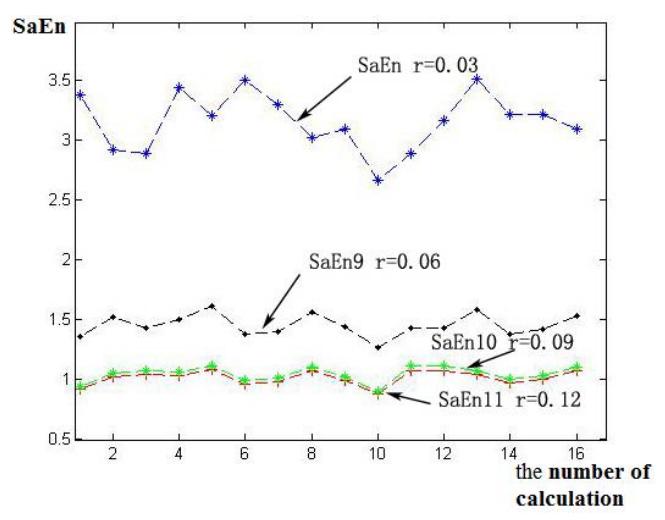

Figure 3 Sample entrop changes with $\mathrm{r}$
It is needed to consider two indexes, namely, computation time and differentiation, for selecting appropriate embedded dimensions $\mathrm{m}$. On the one hand, the real time required fast calculation rate and short calculation time, so $\mathrm{m}$ should be smaller. On the other hand, the differentiation should be larger to well design the quantitative evalution model of technological properties of welding, so the standard deviation should be generally within 0.05 , as well as the selected $\mathrm{m}$ should not be too small. In the following analysis of this paper, $\mathrm{m}$ should be 5 by the overall consideration on two factors.

\subsubsection{Influence of given threshold $r$ on SaEn}

The given threshold $\mathrm{r}$ was determined by the standard deviation, and it was $0.1 \sim 0.5$ times as large as standard deviation. The current signals with better quality were selected, and the SaEn was calculated as $\mathrm{r}$ was $0.03,0.06$, 0.09 and 0.12, respectively. Then, four curves of SaEn8, SaEn9, SaEn10 and SaEn11 were obtained, as shown in Figure 3. Clearly, when $r$ was 0.03 , the SaEn8 curve was extremely unstable, as well as the change amplitude was far more than those of the other two curves. When $r$ was 0.06 , the SaEn9 curve was stable, about 1.5. When $r$ was 0.09 and 0.12 , respectively, SaEn10 and SaEn11 curves almost overlapped, and they were very stable.

Therefore, different $\mathrm{m}, \mathrm{r}$ and $\mathrm{N}$ had larger influence on the calculation results of SaEn. For the double-pulse MIG welding of aluminium alloy, the SaEn distribution of current signals were reasonable as well as the time for calculation was less when $\mathrm{m}$ was $5, \mathrm{r}$ was 0.08 , and $\mathrm{N}$ was 2000 .

\section{Test analysis}

\subsection{Main influence factors of welding quality analysis by test method}

Table 1 Welding condition and process parameters

\begin{tabular}{ccccccl}
\hline $\begin{array}{c}\text { Welding } \\
\text { paramete } \\
\text { rs }\end{array}$ & $\begin{array}{c}\text { Wave } \\
\text { control } \\
\text { mode }\end{array}$ & $\begin{array}{c}\text { Avera } \\
\text { ge } \\
\text { current }\end{array}$ & $\begin{array}{c}\text { Argo } \\
\text { ngas } \\
\text { flow }\end{array}$ & $\begin{array}{c}\text { Wire } \\
\text { feedin } \\
\text { g } \\
\text { speed }\end{array}$ & $\begin{array}{c}\text { Throu } \\
\text { gh the } \\
\text { wire }\end{array}$ & $\begin{array}{l}\text { Welding } \\
\text { speed }\end{array}$ \\
\hline Value & $\begin{array}{c}\text { Trapezoid } \\
\text { al wave }\end{array}$ & $135 \mathrm{~A}$ & $\begin{array}{c}18 \\
\mathrm{~L} / \mathrm{mi} \\
\mathrm{n}\end{array}$ & $\begin{array}{c}4.8 \\
\mathrm{~m} / \mathrm{mi} \\
\mathrm{n}\end{array}$ & $15 \mathrm{~mm}$ & $\begin{array}{l}80 \mathrm{~cm} / \mathrm{m} \\
\text { in }\end{array}$ \\
\hline
\end{tabular}

The AA6061 aluminium alloy was the base metal for the test, and its size was $200 \times 50 \times 4 \mathrm{~mm}$. The welding wire was ER4043 with a diameter of $1.2 \mathrm{~mm}$. Both chemical components were shown in Table 1 . Before welding, the oxide film on the surface of base metal A was polished away, and the oxide film on the surface of base metal B was partly polished away. However, base metal $\mathrm{C}$ was not polished, and the other welding parameters were shown in Table 2.

\subsection{Results and discussion}

MATLAB software was used to calculate the collected current $\mathrm{SaEn}$ in the test process. The SaEn curves of different tests were obtained by connecting sixteen groups of SaEn values when $\mathrm{m}$ was $5, \mathrm{r}$ was 0.08 , and $\mathrm{N}$ was 2000, as shown in Figure 4.

It is clearly shown in Figure 4 that the fluctuation of SaEn12 in test 12 was small, the mean was 0.7327 , and CSaEn was 0.0947. According to the calculation results, it is concluded that the welding quality of test 12 was the best, the welding quality of test 13 was medium, but that of test 14 was worse. Figure 5 showed the welds obtained in three groups of tests. 


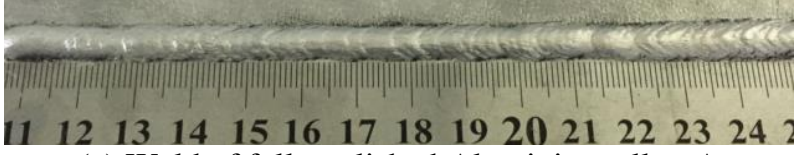

(a) Weld of fully polished Aluminium alloy A

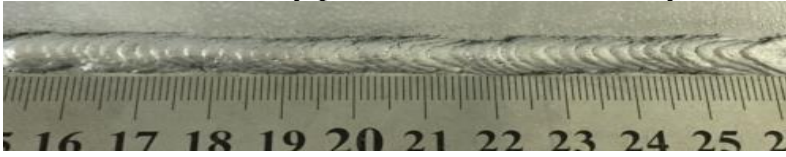

(b) Weld of partial polished Aluminium alloy B Partial

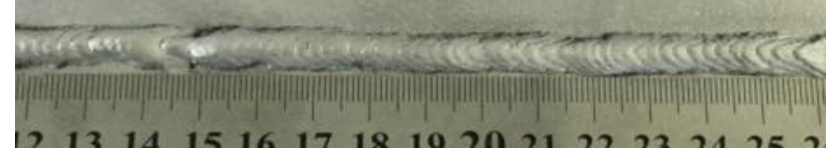

(c) Weld of Aluminium alloy $\mathrm{C}$ with severe oxidation

Figure 5 Weld profiles of different base metal

As shown in Figure 5, the weld surface of test A was smooth, the width was uniform, and there was no spatter on the surface of welds, and the quality of welds were the best. In test B, the width of welds was uneven, and the welding quality was better. However, for test C, there was arc interruption in the welds, the width was larger, and the welding quality was medium. The predicted results were consistent with the practical analysis results.

In short, CSaEn was an effective quantitative analysis index for evaluating welding quality on the premise of the same welding current as well as other matching welding parameters.

\section{Conclusions}

This paper proposed a kind of quantitative analysis method for the welding quality based on CSaEn. It is found that $\mathrm{CSaEn}$ increased with the decrease of the electrical signals stability, which showed the effectiveness of CSaEn for the welding quality analysis. Furthermore, the influences of the bedded dimension m, the given threshold $\mathrm{r}$ and the sample length $\mathrm{N}$ on CSaEn were discussed. When $\mathrm{m}$ was 5 , $\mathrm{r}$ was 0.08 , and $\mathrm{N}$ was 2000, the distribution of CSaEn was reasonable and the calculation time was less.

\section{References}

1. Pincus S M. Approximate entropy as a measure of system complexity[J]. Proceedings of the National Academy of Sciences of the United States of America, 1999, 88(6): 2297-2301

2. Zhang weichao, Yang lijun, lu xiaoqing. Research on adaptive control of aluminum alloy p-mig welding based on approximate entropy measurement [J]. Journal of physics, 2011, 60(02): 152-160

3. Nie Jing, Shi Yu, jian-kang huang, et al. P aluminum alloy MIG welding stability of approximate entropy of neural network prediction $[\mathrm{J}]$. Journal of welding, 2010, 31 (8) : 77-80 + 116-117

4. Tolle C R, Laviolette R A, Smartt H B, et al. Is there evidence of determinism in droplet detachment within the gas metal arc welding process[C]. $6 \mathrm{TH}$ International conference on Trends in Welding Research. APRIL, 2002: 15-19

5. Richman J S, Moorman J R. Physiological time-series analysis using approximate entropy and sample entropy[J]. AMERICAN JOURNAL OF PHYSIOLOGY-HEART AND CIRCULATORY PHYSIOLOGY, 2000, 278(6): 2039-2049

6. Li huan, wang junhong, ai danfeng, et al. Multi-information synchronization acquisition system in arc welding process [J]. Journal of tianjin university, 2012, 45(2): 184-189 